\title{
Estimation of Fluctuation Characterizations by USV-Operation Simulations in Sea State 3
}

\author{
Jae-Han Park, Ji-Hun Bae, and Moon-Hong Baeg \\ Robotics ReD Group, Korea Institute of Industrial Technology (KITECH), Ansan, Republic of Korea \\ Correspondence should be addressed to Jae-Han Park; hans1024@kitech.re.kr
}

Received 5 July 2017; Revised 22 September 2017; Accepted 12 October 2017; Published 6 November 2017

Academic Editor: Aiguo Song

Copyright (c) 2017 Jae-Han Park et al. This is an open access article distributed under the Creative Commons Attribution License, which permits unrestricted use, distribution, and reproduction in any medium, provided the original work is properly cited.

\begin{abstract}
This paper proposes a method based on simulation techniques for fluctuation characterizations of unmanned surface vehicle (USV) operations under Sea State 3. In order to simulate the operations of a USV in Sea State 3, we generated the data of sea surfaces using linear wave theory and utilized the motion equation. Fluctuation analysis results by the proposed simulation method could provide crucial information for designing the stabilization system for the critical equipment on a USV. Through these works, it was verified that the design specifications such as range of motion, maximum speed, and acceleration could be estimated using the simulation data.
\end{abstract}

\section{Introduction}

An unmanned surface vehicle (USV) refers to an object that moves autonomously or is remotely operated on the sea surface without requiring crewmen [1]. It was initially developed for military purposes in the 1940s. Subsequently, the application of USVs has expanded to a wide range of fields including surveillance and reconnaissance, port surveillance, and maritime surveys. Specifically, USVs are typically used for tasks that were previously performed by manned ships (such as mine hunting and elimination and espionage into enemy territory), dangerous tasks involving neritic regions that are challenging to access using manned ships, or polluted sea areas. They are also popular as being highly useful in tasks wherein crewmen's exhaustion constitutes an issue during ocean surveillance, maritime surveys, and other tasks involving extended hours. Although USVs were primarily used for military purposes, the demand for their public use has increased recently.

In several cases, special equipment is provided on the USV to enable its utilization for various purposes. However, most of the equipment requires attention during operation, and safety with respect to disruptions from USV motions must be considered. Recently, the operational conditions of
USVs were in general set to Sea State 3. Hence, it is extremely important to assess the characteristics of disruptions that are applied to USVs through the influence of waves at the maximum operational speed and the sea state, to stabilize these conditions $[2,3]$. For this purpose, it is necessary to obtain information about the fluctuation characteristics in the specified sea state condition. However, it is highly challenging to obtain such characteristic information through measurement in actual environments. In actual environments, the sea state condition is beyond control; therefore, obtaining characteristic information can be performed only when the sea state condition is favorable. Moreover, as the fluctuation characteristics vary with respect to the shape of the ship, only the data acquired and analyzed by a similar type of vessel will be valid. It is also challenging to collect fluctuation characteristics data for various operating conditions because the fluctuation characteristics are likely to vary according to the USV operating conditions. That is, the measurement data for analyzing the fluctuation characteristics with respect to the sea state are available only when the sea state, type of vessel, and operating conditions are satisfied.

Simulation-based methodologies can overcome such problems in data acquisition in real-world environments [2]. In simulation environments, the sea state can be regulated 


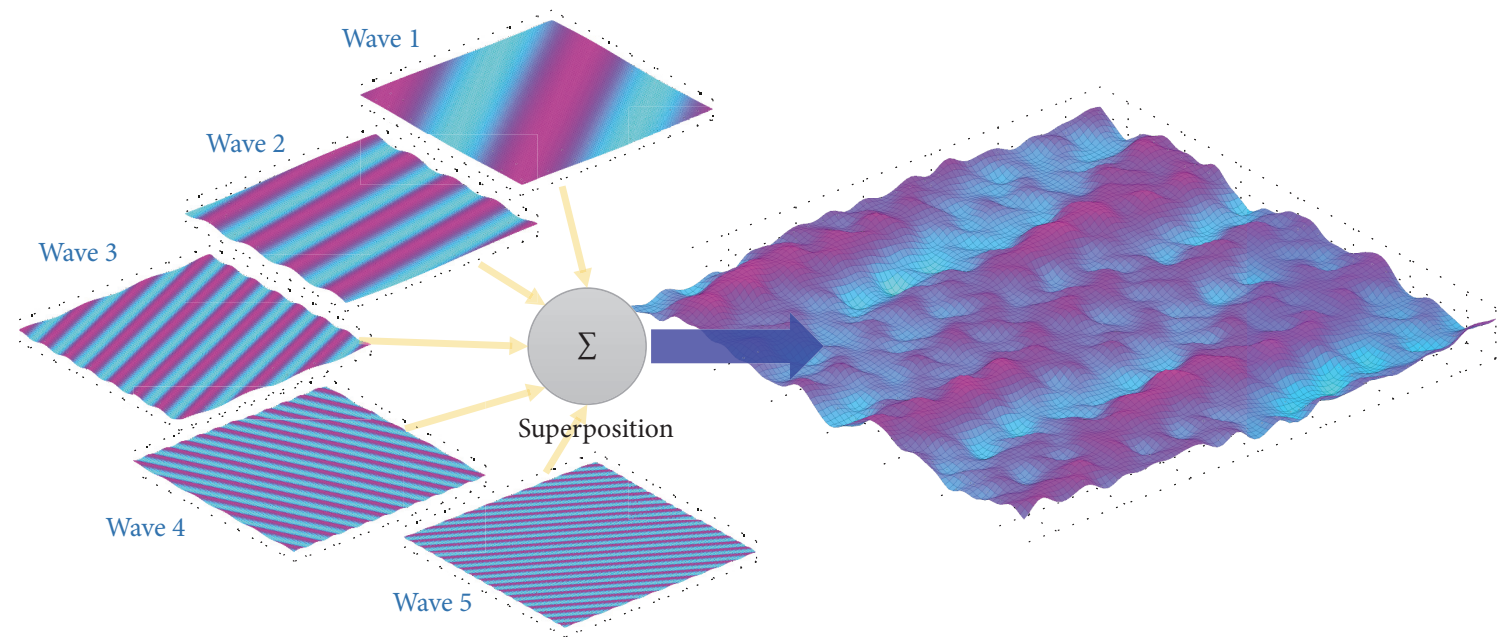

FIGURE 1: Representation of a sea surface generated by superposing linear waves.

freely, and the type of vessel and operating conditions can be defined as desired. Therefore, this study proposes a method to estimate the characteristics with respect to the fluctuations and disturbances that occur during a USV-operation simulation at Sea State 3 conditions. In the method of generating data through simulation, the adequacy of the USV simulation model for the actual motions in actual environments is directly related to the reliability of the acquired data. The surface of the sea can be configured based on the sea state by synthesizing waves that are proposed using linear wave theory [4-7]. USV-operation simulations can be accomplished by estimating the pose of the USV using the sea surface information that is generated according to the specific sea state condition and calculating the motion of the USV according to the operation conditions. USV-motion simulations are performed, and the location and direction are calculated and collected to assess the characteristics of the fluctuations and disturbances that are applied on the USV.

The rest of the paper is organized as follows: Section 2 introduces the linear wave theory and the simulation for Sea State 3 that uses the theory. Section 3 explains the method of estimating the location and direction of the USV based on the form of the sea surface and discusses the USV-motion model that was applied. Section 4 introduces the fluctuation and disturbance data of the USV that was obtained through an actual simulation. Subsequently, in the conclusion, a plan for utilizing the study results and directions for future development is presented.

\section{Generation of Sea Surface Data for Sea State 3}

2.1. Linear Wave Theory. This theory is a method that replicates the ocean through simulation and generates data on the sea surface by synthesizing waves $[4,6]$. The sea state can be reproduced through this method; thus, ship motions are simulated accordingly, and their characteristics are also analyzed. In linear wave theory, the surface of the sea with respect to the sea state is expressed through a linear superposition of waves with varying frequencies in (1), as shown in Figure 1.

$$
\begin{aligned}
& \varsigma(x, y)=\sum_{i=1}^{\infty} A_{i} \\
& \quad \cdot \sin \left(\omega_{i} t-k_{i} x \cos \left(\theta_{i}(t)\right)-k_{i} y \sin \left(\theta_{i}(t)\right)+\varepsilon_{i}(t)\right) .
\end{aligned}
$$

Here, $A_{i}$ denotes the amplitude of the $i$ th wave, $\omega_{i}$ denotes each individual frequency, $k_{i}$ denotes the wave number, and $\theta_{i}$ and $\varepsilon_{i}$ denote the direction and phase, respectively, of the waves. Additionally, $\theta_{i}$ and $\varepsilon_{i}$ are typically modeled as random variables that are distributed between 0 and $2 \pi$.

Equation (1) includes random internal variables. The sea surface data that expresses this also corresponds to a random variable. The wave height $H$ with respect to the random sea surface distribution is approximately modeled using the Rayleigh distribution as follows:

$$
f(H)=\frac{H}{4 m_{0}} \cdot \exp \left(-\frac{H^{2}}{8 m_{0}}\right),
$$

where $m_{0}$ denotes the parameter that is related to the mean height

$$
H_{m}=\int_{0}^{\infty} H \cdot f(H) d h=\sqrt{2 \pi m_{0}} .
$$

The significant wave height is a highly critical parameter that is related to the sea state. It refers to the average height of the waves, which corresponds to $1 / 3$ of the maximum height of all the waves that occur within a certain time period as follows:

$$
H_{1 / 3}=\int_{H_{0}}^{\infty} H \cdot f(H) d h
$$


TABLE 1: Characteristics data for Sea States 1-5.

\begin{tabular}{lcc}
\hline Sea state & Significant wave height $[\mathrm{m}]$ & Description \\
\hline 1 & $0-0.1$ & Calm \\
2 & $0.1-0.5$ & Smooth \\
3 & $0.5-1.25$ & Slight \\
4 & $1.25-2.5$ & Moderate \\
5 & $2.4-4.0$ & Rough \\
\hline
\end{tabular}

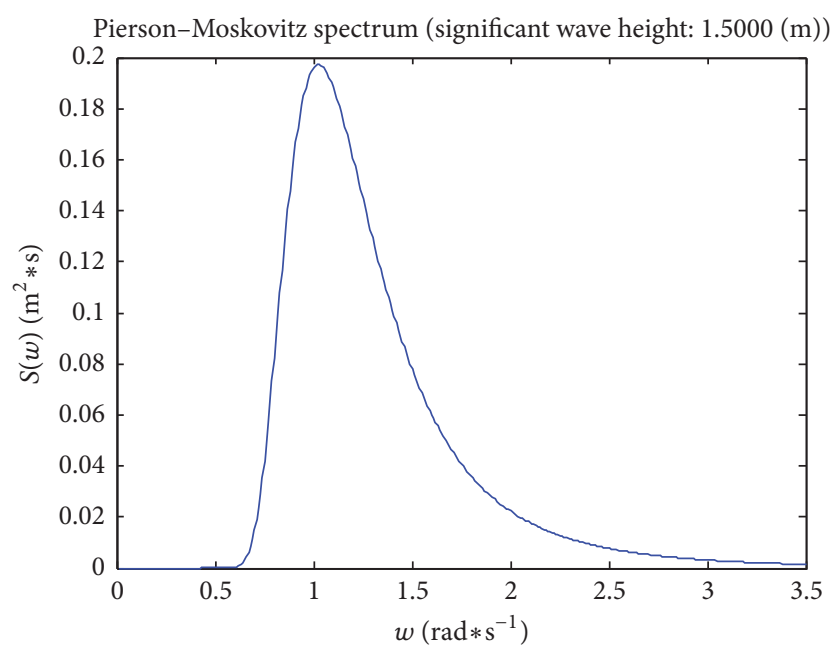

Figure 2: Pierson-Moskovitz wave spectrum for $H_{1 / 3}=1.5[\mathrm{~m}]$.

Here, $H_{0}$ is defined as

$$
\int_{H_{0}}^{\infty} f(H) d h=\frac{1}{3}
$$

The wave spectrum is another critical factor related to sea surface modeling. It represents the energy distribution in the frequency range with respect to the waves that constitute the surface of the sea. It proposes a mathematical model as per the ITTC 1978 and Pierson-Moskovitz models. The Pierson-Moskovitz spectrum model [9] is expressed in (6), and Figure 2 shows the distribution of the wave spectrum with respect to the significant wave height $H_{1 / 3}=1.5[\mathrm{~m}]$.

$$
S(j \omega)=\frac{8.1 \times 10^{-3} g^{2}}{\omega^{5}} \exp \left(-\frac{0.0323 \times g^{2}}{\left(H_{1 / 3}\right)^{2} \times \omega^{5}}\right) .
$$

2.2. Sea State 3 Simulation. Critical data that defines the sea state includes parameters such as significant wave height, significant range of periods, average period, and wind speed. Table 1 presents the characteristic data of Sea States 1-5 for the Pierson-Moskovitz wave spectrum model [10].

Based on the linear wave theory, the waves synthesized in the wave spectrum must be sampled by frequency prior to generating data on the surface of the sea. Based on Table 1, the significant wave height at Sea State 3 corresponds to 1.2192 [m] (4 feet). Hence, the wave spectrum distribution was generated through (6). Subsequently, data at 2-7 [s] (which corresponds to the significant range of periods at Sea State 3) were utilized to obtain a balanced sample of 30 waves that are relevant to $\omega_{1}-\omega_{30}$ within a range of $0.8975-3.1415[\mathrm{rad} / \mathrm{s}]$. Thus, it is relevant to this section in the frequency range. Therefore, $\Delta \omega$ corresponds to $0.0748[\mathrm{rad} / \mathrm{s}]$ in this case. Figure 3 shows the Pierson-Moskovitz wave spectrum distribution for Sea State 3 and 30 sampled waves $\left(\omega_{1}-\omega_{30}\right)$.

In order to generate sea surface data, the amplitude $A_{i}$ with respect to the waves of $\omega_{i}$ that are sampled through (1) can be obtained as follows:

$$
A_{i}=\sqrt{2 S\left(\omega_{i}\right) \cdot \Delta \omega}
$$

The wave number $k_{i}$ of the $i$ th wave and the relevant $\omega_{i}$ are as follows:

$$
k_{i}=\frac{2 \pi}{\lambda_{i}}=\frac{\omega_{i}^{2}}{g} .
$$

The direction $\theta_{i}$ and phase $\varepsilon_{i}$ of each wave are random variables, and, thus, the initial values were sampled through random balanced distribution variables defined from $0-2 \pi$. Thereafter, they were implemented using the random walk process, which constantly changes with time in a random manner within a marginal range.

Figure 4 shows the results of the sea surface simulation by using (1) with the 30 sampled waves, for Sea State 3. It was generated in an area of $150[\mathrm{~m}]$ in each of $x$ and $y$ directions on the surface of the earth. The results of estimating the form of the sea surface that changes with time and its characteristics data were similar to the data for Sea State 3. Hence, this verified the validity of the process of estimating and analyzing USV fluctuation characteristics through a simulation of Sea State 3 by using linear wave theory.

\section{Estimation of USV Motion at Sea}

3.1. Estimation of USV Position. All ships operated at sea, including USVs, involve six degrees of freedom (DOFs) in motion $[8,11,12]$. Three of these DOFs involve motion regarding location, while the other three involve directional motion. As shown in Figure 5, motions involving a ship's location are defined as surging (backward and forward), swaying (left and right), and heaving (up and down). Additionally, motions regarding direction are defined as rolling (spinning forward 


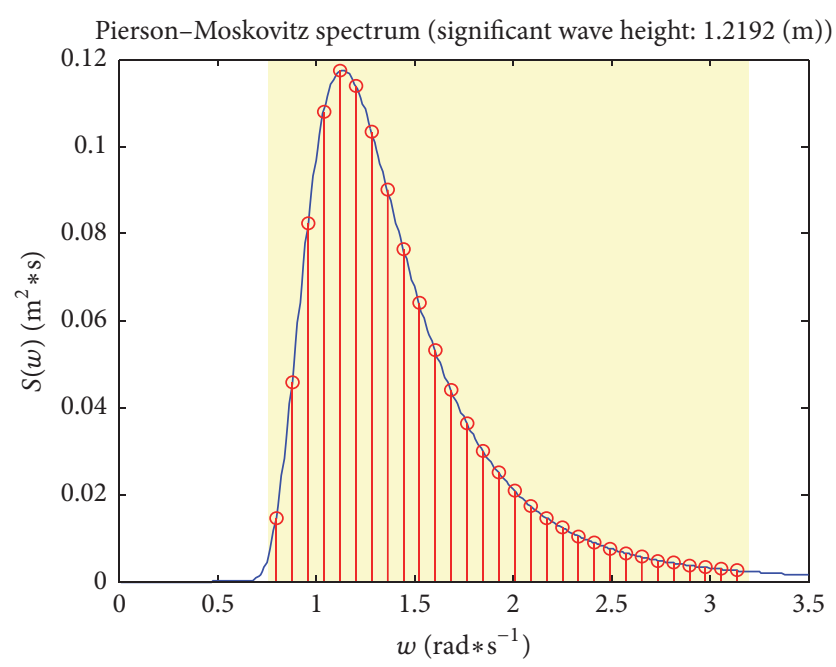

Figure 3: Wave spectrum curve for Sea State 3.

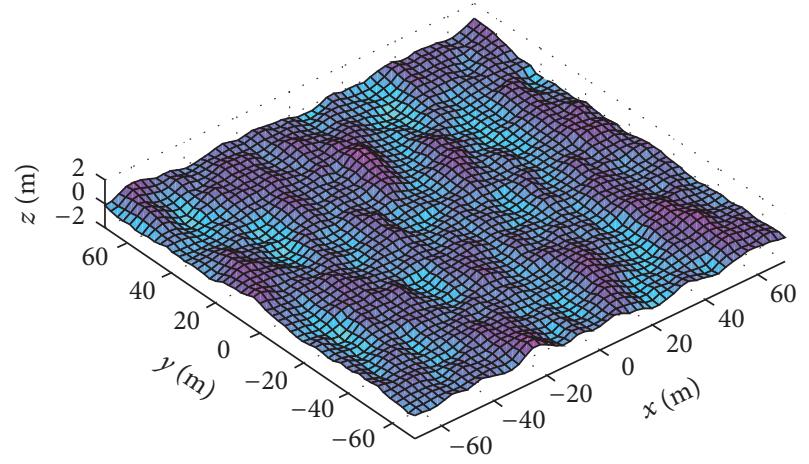

FIGURE 4: Simulation result with the 30 sampled waves for Sea State 3.

and backward), pitching (spinning up and down), and yawing (spinning left and right).

With respect to the operation of an USV, elements that are affected through disturbances from the sea state correspond to heaving motions (related to the location and rolling) and pitching motions (related to direction). Thus, these three motion elements with respect to the surface of the sea are estimated. Response surface methodology (RSM) $[13,14]$ was used in this study to obtain a detailed estimate of the USV's location and direction with respect to the surface of the sea. The RSM requires a finite number of measuring points. The points that are affected by the surface of the sea owing to the buoyancy of the USV were approximated as an ellipse, as shown in Figure 6, and selected as the measuring points. The measuring points were determined under the assumption that the length and width of the USV with respect to the test corresponded to $6[\mathrm{~m}]$ and $3[\mathrm{~m}]$, respectively.

The pose estimation of the USV using RSM is performed through the following process: First, data on the surface of the sea are calculated by using (1) with respect to the 24 measuring points shown in Figure 6. Next, least squares regression is performed to obtain a plane equation that relates to the measuring points and the data on the surface of the sea with

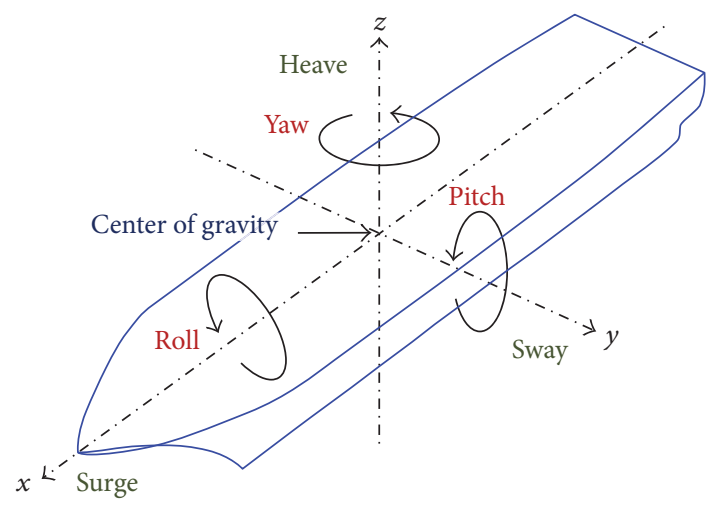

Figure 5: Six-DOF vessel motions [8].

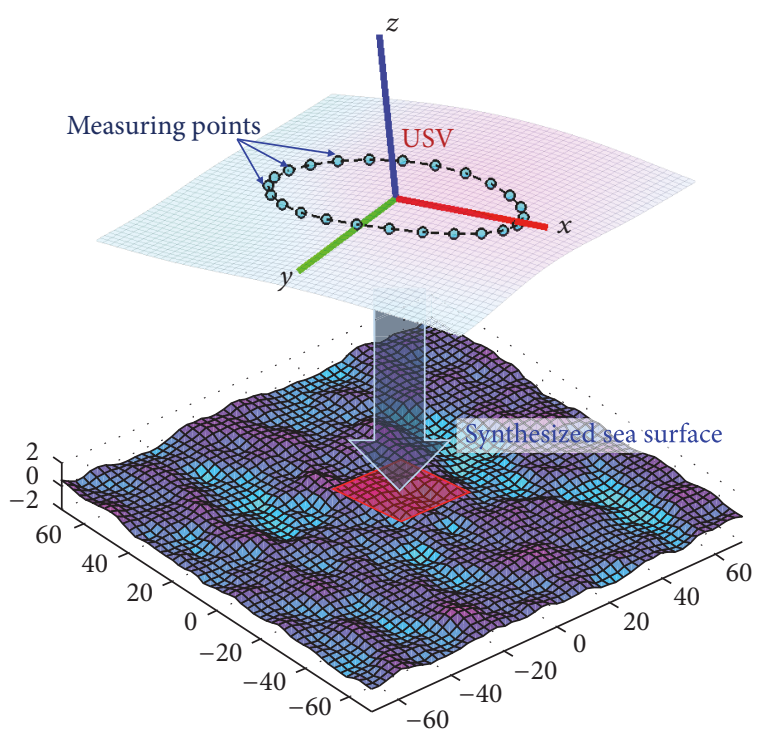

FIGURE 6: Measuring points for pose estimation of USV.

respect to these points. The center of gravity for the measured data corresponds to the USV's estimated location data. The plane's normal vector data is used to estimate the USV's roll and pitch direction. Figure 7 shows the USV's pose and motion that were estimated by using this method. The red line in the figure denotes the normal vector estimated by using RSM. The red line denotes the gradient vector with respect to the direction of gravity. An increase in size is likely to result in the occurrence of a disturbance in the yaw direction owing to gravity. However, this influence was not considered in this study. The data on the surface of the sea from the simulation results was used to verify as to whether the USV's pose and motion data estimated using RSM were naturally expressed.

3.2. USV-Motion Model. With respect to the USV's six-DOF motion, the directional information on the location based on the $z$-axis (up and down direction), rolling, and pitching was estimated using the data on the surface of the sea. The data on 


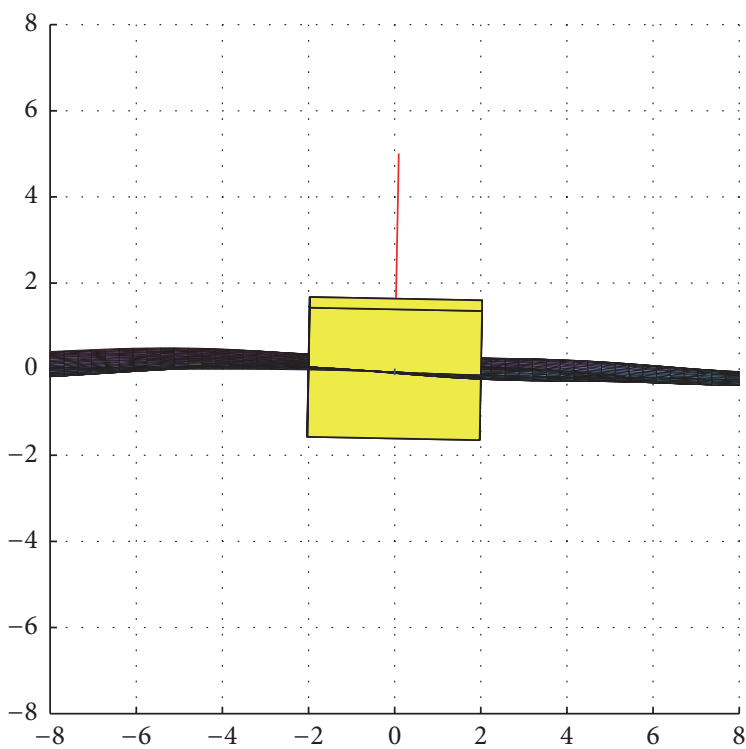

(a)

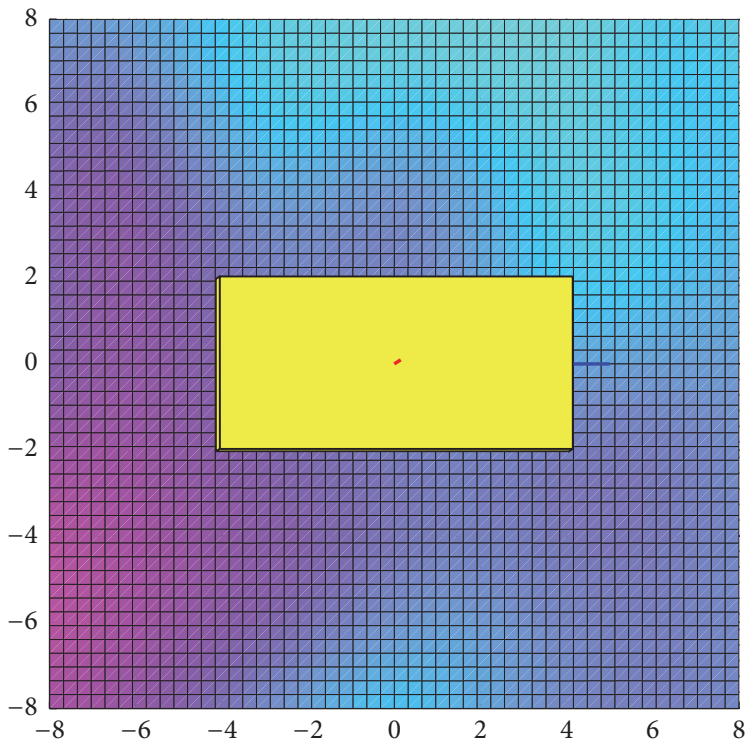

(c)

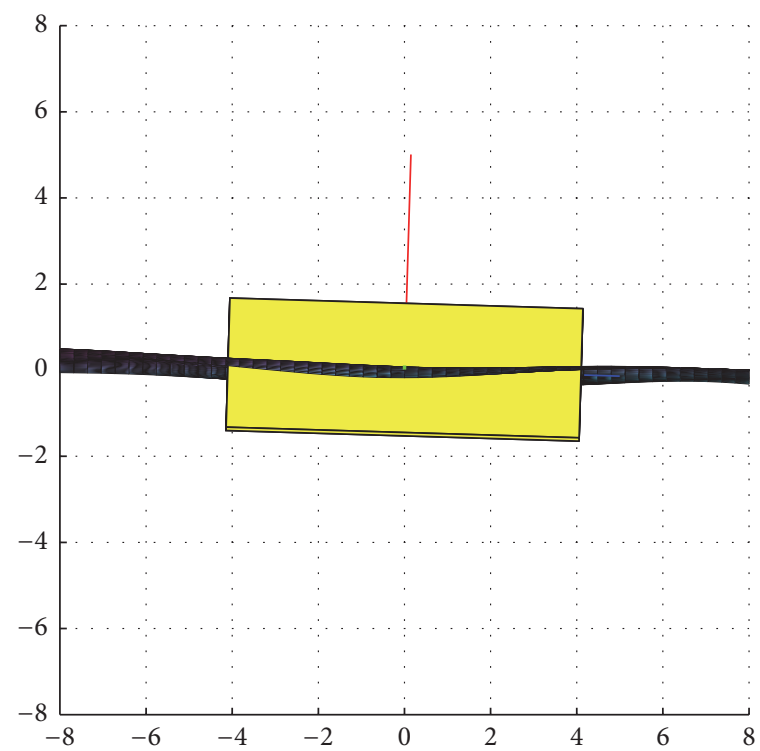

(b)

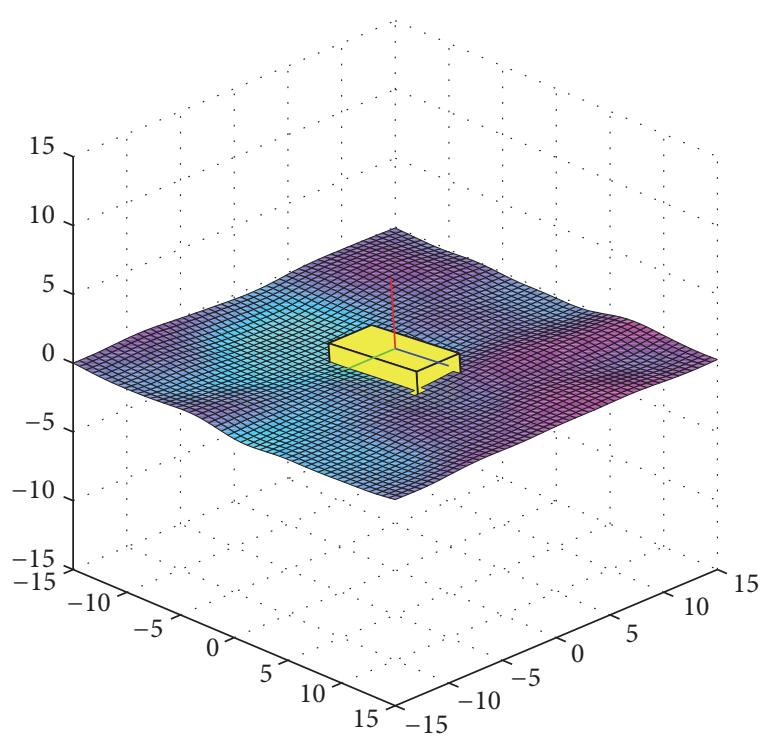

(d)

FIGURE 7: Estimation of six-DOF motion of USV with respect to the sea surface data by using RSM. (a) View of $y$ - $z$ plane direction; (b) view of $x-z$ plane direction; (c) view of $x-y$ plane direction; (d) view of azimuth $=45\left[^{\circ}\right]$ and elevation $=25\left[^{\circ}\right]$.

the remaining three motions (i.e., location on the $x$ - and $y$ axes and yaw data, which reveals the motion from left to right) are significantly influenced by the USV's driving system; thus, they were calculated using the USV-motion model. Rotation speed $\omega_{k}$, which varies the direction and speed $v_{k}$ with respect to the direction of progress, is input into the USV drive system. The output factors that are influenced by the same correspond to the $x$ and $y$ locations and yaw angle $\theta_{k}$ and are obtained using the difference equation as expressed by

$$
\mathbf{x}_{k+1}=\boldsymbol{\Phi} \mathbf{x}_{k}+\Delta t \mathbf{H}_{k} \mathbf{u}_{k}
$$

where

$$
\begin{aligned}
\mathbf{x}_{k} & =\left[\begin{array}{l}
x_{k} \\
y_{k} \\
\theta_{k}
\end{array}\right], \\
\mathbf{u}_{k} & =\left[\begin{array}{c}
v_{k} \\
\omega_{k}
\end{array}\right], \\
\mathbf{\Phi} & =\mathbf{I}, \\
\mathbf{H}_{k} & =\left[\begin{array}{cc}
\cos \left(\theta_{k}\right) & 0 \\
\sin \left(\theta_{k}\right) & 0 \\
0 & 1
\end{array}\right] .
\end{aligned}
$$




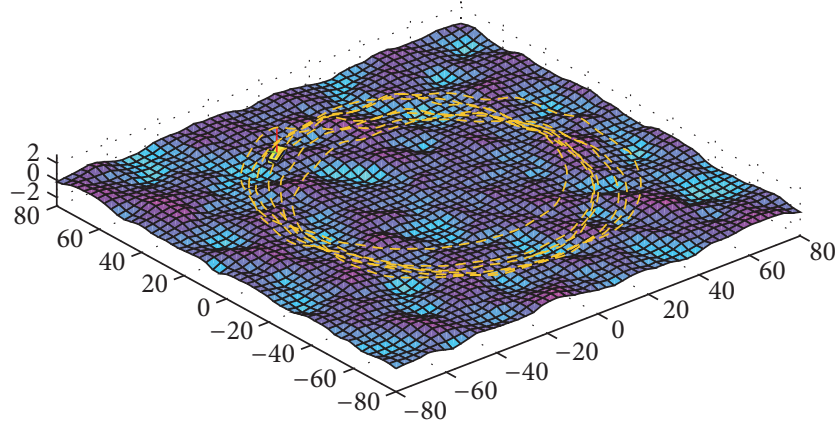

FIGURE 8: USV trajectory for a simulation under Sea State 3.

The above equation was used to generate the surface of the sea by simulating Sea State 3 . The USV pose estimation method was used in a similar manner to estimate the USV's motions with respect to a random direction of progress $v_{k}$ and rotation speed $\omega_{k}$. These were used to predict disturbance characteristics such as fluctuation that occur during operation. The following section analyzes the characteristics of the fluctuations that occur during USV operation based on the USV simulation results at Sea State 3.

\section{USV Simulation under Sea State 3}

In order to assess the characteristics of the fluctuations during the operation of a USV, a relatively high speed $v=35$ [Knots] and rotation speed $\omega=0.35[\mathrm{rad} / \mathrm{s}]$ were applied. The simulation was performed for $60[\mathrm{~s}]$. Figure 8 shows the screen depicting the USV trajectory for a simulation at the surface of the sea at Sea State 3. The yellow box denotes the USV; the simulations at various conditions are conducted by applying random $v$ and $\omega$ values.

Using the heave, roll, and pitch motion data that are collected by simulations, we estimated the frequency characteristics of the fluctuation of USV motion through the fast Fourier transform. Figure 9 shows the data of roll and pitch motions collected through simulations. Figure 10 shows the frequency spectrum for the collected roll and pitch data. The maximum fluctuation that occurred during operation at $v=35$ [knots] and $\omega=0.35[\mathrm{rad} / \mathrm{s}]$ under Sea State 3 was observed as approximately $2.5-3[\mathrm{~Hz}]$ in the rolling and pitching directions. Therefore, the stabilization system in a USV that is operated under these conditions should absorb these fluctuation disturbances, and the actuator responsiveness in a dynamic system must significantly exceed the fluctuation disturbances.

Additionally, the design specifications of the stabilization system comprising the operating range, maximum speed, and acceleration could be determined based on the collected USV-motion data. In particular, the active stabilization system generates a motion counter to the fluctuation of the USV such that the external disturbance does not affect the equipment mounted on the USV. The active stabilization system must be designed to operate more rapidly than the motion due to external disruptions to compensate for the fluctuations of the USV. Therefore, the proposed USVoperation simulations were performed in order to estimate the extent of external fluctuation disruptions.

To cover all feasible situations, we performed 100 USVoperation simulations for various $v$ and $\omega$ values to measure the range of position, velocity, and acceleration for roll, pitch, and heave motions. The information constituting the estimated range of position is used to determine the operating range of the stabilization system. The information on the range of velocity and acceleration is also critical for determining the specifications of the maximum speed and torque of the actuators in the active stabilization system.

Figure 11 shows the measured values of the maximum position, velocity, and root mean square (RMS) acceleration for roll, pitch, and heave motions in 100 USV-operation simulations. Based on that information, we estimated the extent of fluctuation that occurred in USV operations. Table 2 presents the range of velocity and acceleration for roll, pitch, and heave motions derived from the results of the 100 operation simulations. Thus, the active stabilization system of the USV under Sea State 3 should be designed to compensate the motion specifications presented in Table 2.

\section{Conclusions}

This study proposed a simulation-based process that derives target specifications required in the stabilization system of a USV for the design of special mission equipment under specified sea conditions. The simulation-based method has numerous advantages over the method of collecting data by actual experiment; however, the adequacy of computation in the simulation affects the reliability of the acquired data. To achieve reasonable reliability, the sea environment was replicated for the specified sea state using a linear superposition of waves proposed by linear wave theory. The motion of a USV in that sea environment was generated by estimating the pose of the USV using the sea surface information and calculating the differential equation according to the operational conditions.

The developed simulator was used to conduct a simulation at the maximum USV speed under Sea State 3, which is the subject of this study. In order for the collected data to contain all feasible situations, 100 USV-operation simulations were performed for various operational conditions under Sea State 3. Using the data obtained in the numerous operation simulations, we could estimate the fluctuation characteristics, which are critical specifications for designing the stabilization controller. Moreover, the operating range, maximum speed, acceleration, and other crucial specifications for designing the active stabilization system could be deduced based on the data collected through the simulations.

\section{Conflicts of Interest}

The authors declare that there are no conflicts of interest regarding the publication of this paper. 


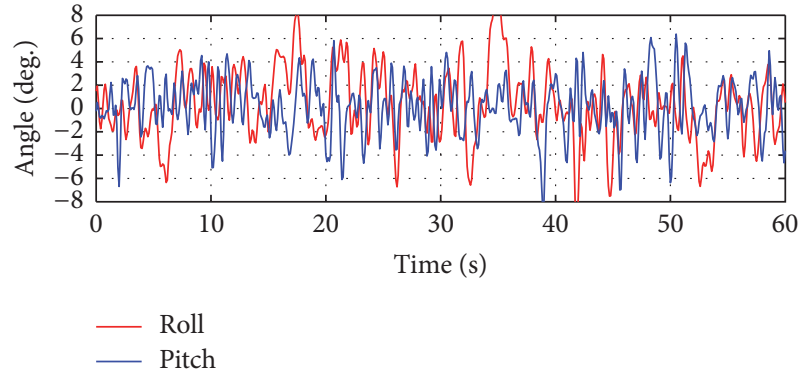

(a)

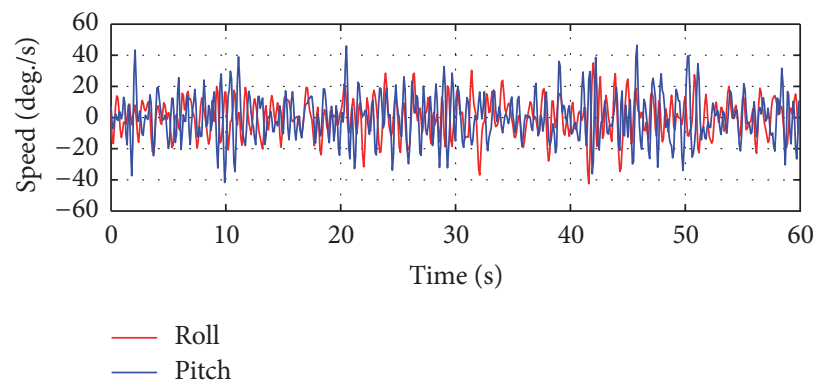

(b)

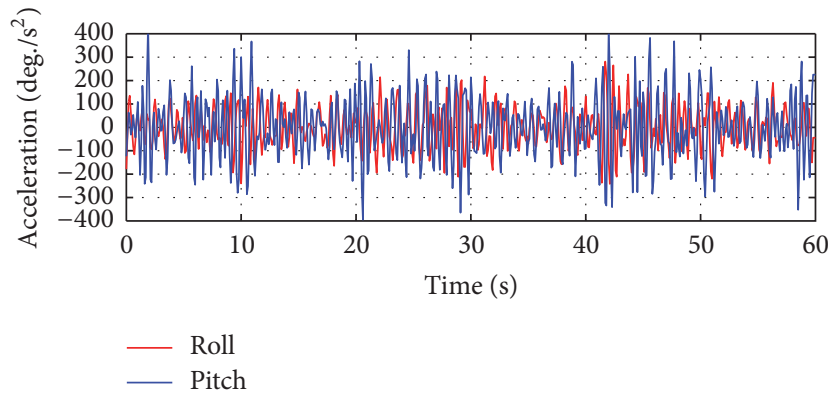

(c)

FIGURE 9: Estimation results of position, speed, and acceleration for roll and pitch motions. (a) Position; (b) speed; (c) acceleration.

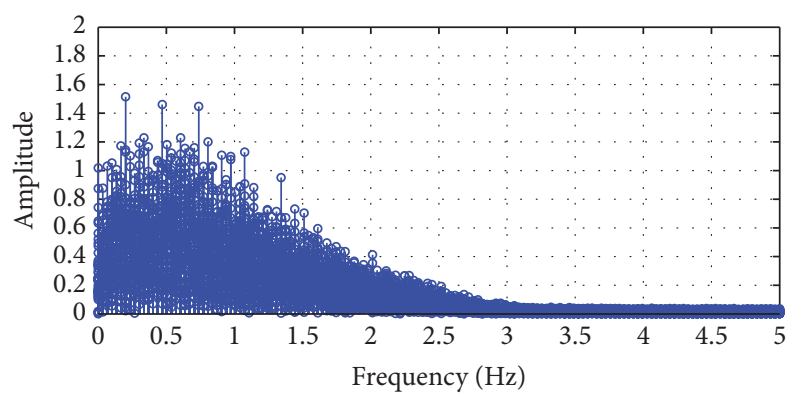

(a)

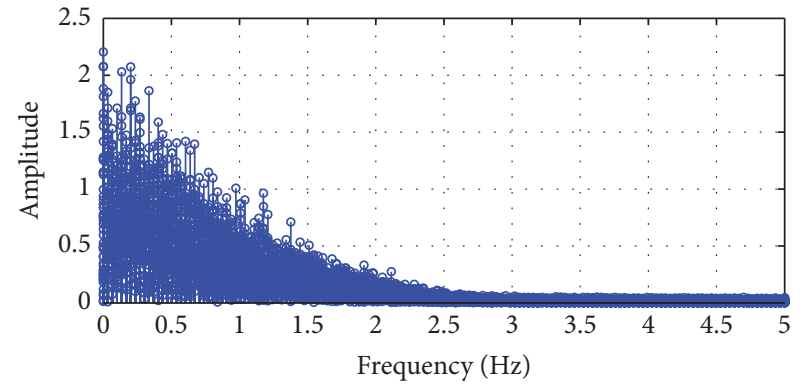

(b)

FIGURE 10: Frequency spectrums of the collected motion data through USV-operation simulations under Sea State 3. (a) Roll motion; (b) pitch motion.

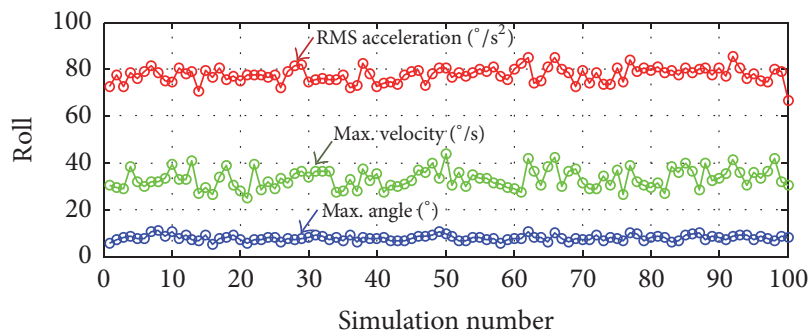

(a)

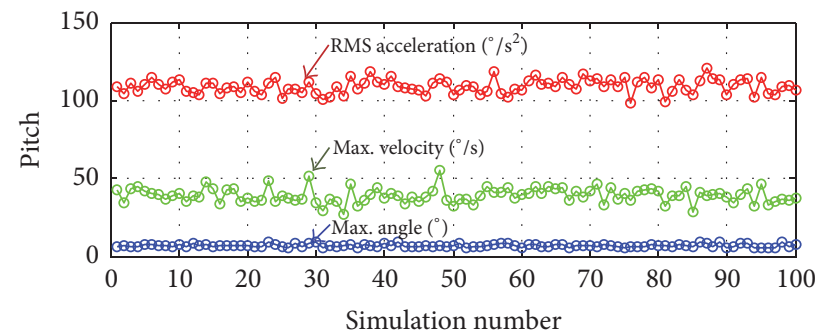

(b)

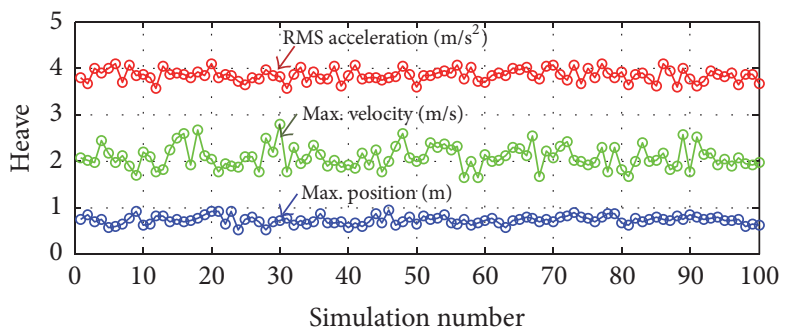

(c)

FIGURE 11: Graphs for maximum position, velocity, and RMS acceleration of USV roll/pitch/heave motion through 100 operation simulations. (a) Roll motion; (b) pitch motion; (c) heave motion. 
TABLE 2: Design specifications for the stabilization system.

\begin{tabular}{lccc}
\hline & Roll & Pitch & Heave \\
\hline Operating range & $\pm 10\left[{ }^{\circ}\right]$ & $\pm 12\left[{ }^{\circ}\right]$ & $\pm 1[\mathrm{~m}]$ \\
Maximum speed & $45[\% / \mathrm{s}]$ & $55\left[0^{\circ} / \mathrm{s}\right]$ & $3[\mathrm{~m} / \mathrm{s}]$ \\
Acceleration (RMS) & $90\left[\mathrm{~s}^{2}\right]$ & $120\left[\mathrm{~s}^{2}\right]$ & $4.5\left[\mathrm{~m} / \mathrm{s}^{2}\right]$ \\
\hline
\end{tabular}

\section{Acknowledgments}

This research was financially supported by the Defense Acquisition Program Administration (DAPA) and the Agency for Defense Development (ADD) of Korea (UC150005DD).

\section{References}

[1] "Unmanned surface vehicle," Wikipedia, the free encyclopedia. https://en.wikipedia.org/wiki/Unmanned_surface_vehicle.

[2] A. Thakur and S. K. Gupta, "Real-time dynamics simulation of unmanned sea surface vehicle for virtual environments," Journal of Computing and Information Science in Engineering, vol. 11, no. 3, Article ID 031005, 2011.

[3] K. Jørgensen, State estimation with wave filtering for an unmanned surface vehicle: by utilizing acceleration feedback, MS thesis, Institutt for Teknisk Kybernetikk, 2011.

[4] M. K. Ochi, Ocean Waves: The Stochastic Approach, Cambridge University Press, Cambridge, UK, 1998.

[5] L. H. Holthuijsen, Waves in Oceanic and Coastal Water, Cambridge University Press, 2010.

[6] L. E. Borgman, "OCean wave simulation for engineering design," Tech. Rep. HEL-9-13, Hydraulic Engineering Lab, California University, Berkeley, calif, USA, 1967.

[7] M. Arhan and R. O. Plaisted, "Non-linear deformation of sea wave profiles in intermediate and shallow water," Oceanologica Acta, vol. 4, no. 2, pp. 107-115, 1981.

[8] "Vessel Motion Calculator (displacements velocities accelerations)," http://www.calqlata.com/productpages/00059-help.html.

[9] W. J. Pierson and L. Moskowitz, "A proposed spectral form for fully developed wind seas based on the similarity theory of S. A. Kitaigorodskii," Journal of Geophysical Research: Atmospheres, vol. 69, no. 24, pp. 5181-5190, 1964.

[10] "Sea state," Wikipedia, the free encyclopedia. https://en.wikipedia.org/wiki/Sea_state.

[11] A. R. J. M. Lloyd, Seakeeping: Ship Behavior in Rough Weather, John Wiley \& Sons, 1989.

[12] A. Biran and R. Lopez-Pulido, Ship Hydrostatics and Stability, Elsevier, 2nd edition, 2014.

[13] K. Marti, Stochastic Optimization Methods, Springer, Berlin, Heidelberg, Germany, 3rd edition, 2008.

[14] R. H. Myers, D. C. Montgomery, and C. M. Anderson-Cook, Response Surface Methodology: Process and Product in Optimization Using Designed Experiments, John Wiley \& Sons, 3rd edition, 2009. 


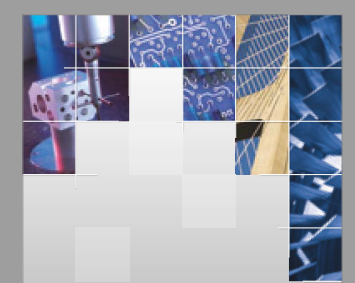

\section{Enfincering}
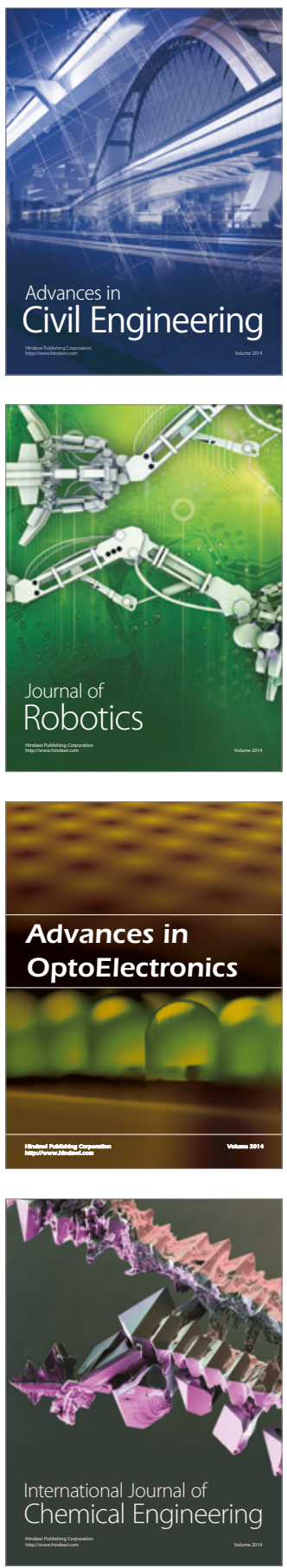

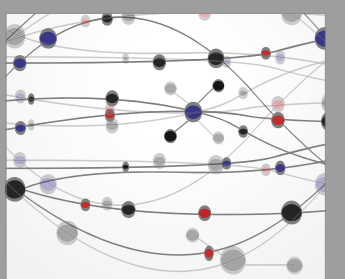

The Scientific World Journal

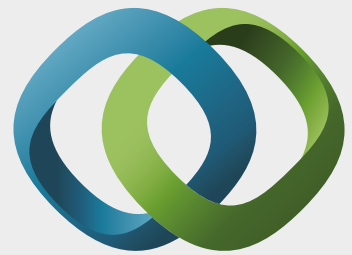

\section{Hindawi}

Submit your manuscripts at

https://www.hindawi.com
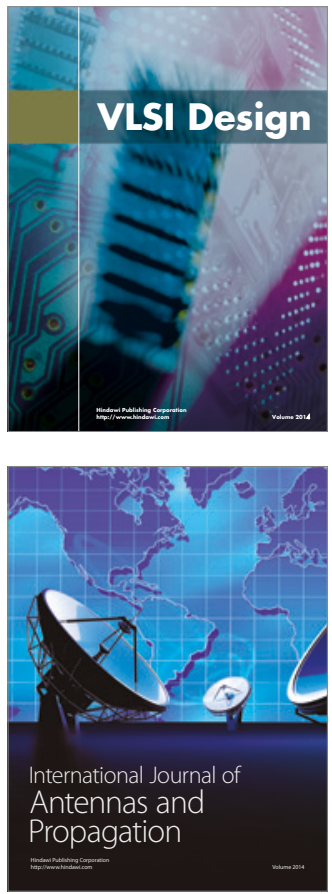

\section{Rotating}

Machinery
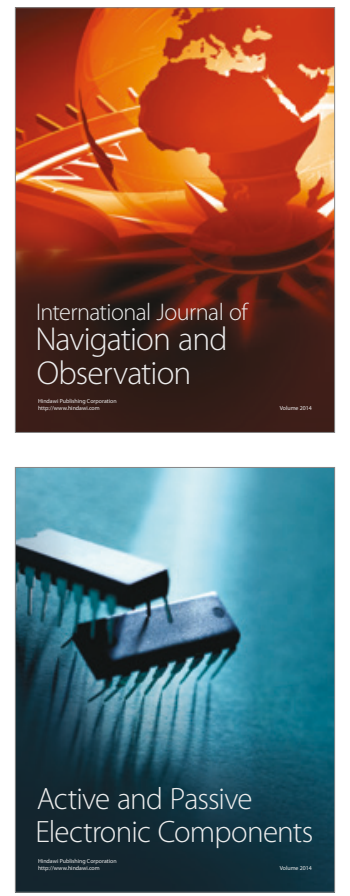
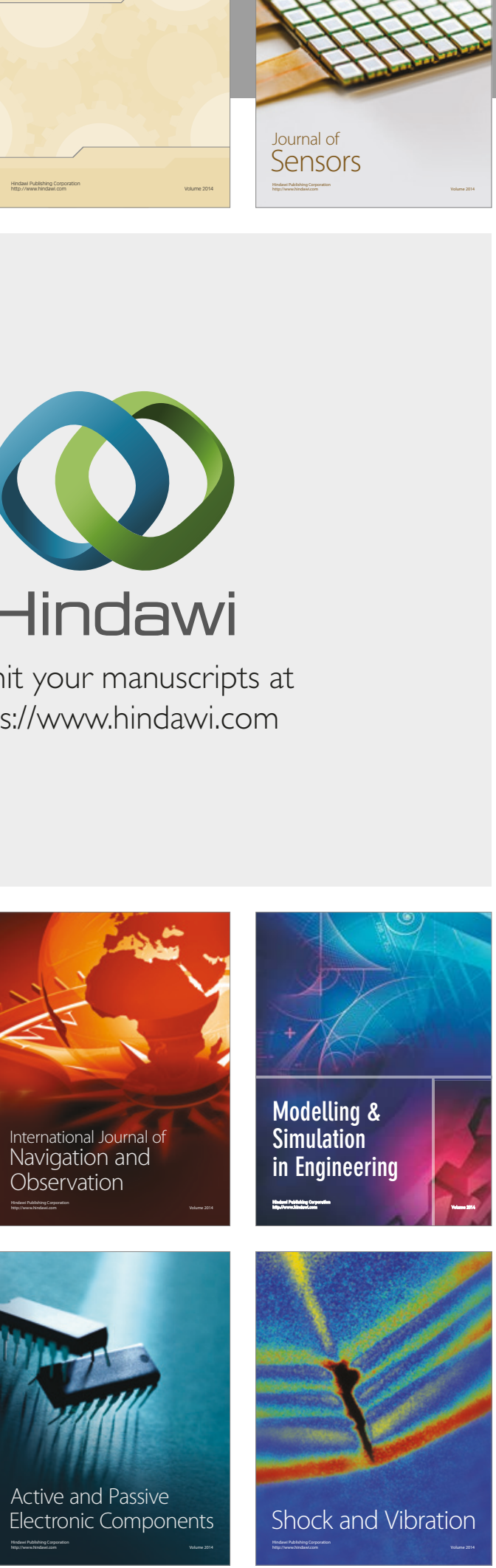
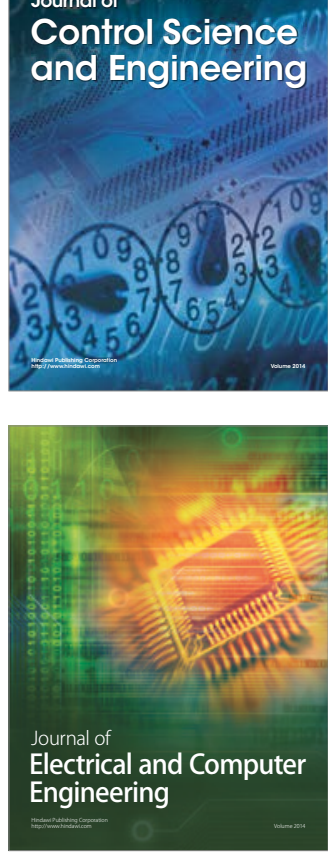

Distributed

Journal of

Control Science

and Engineering
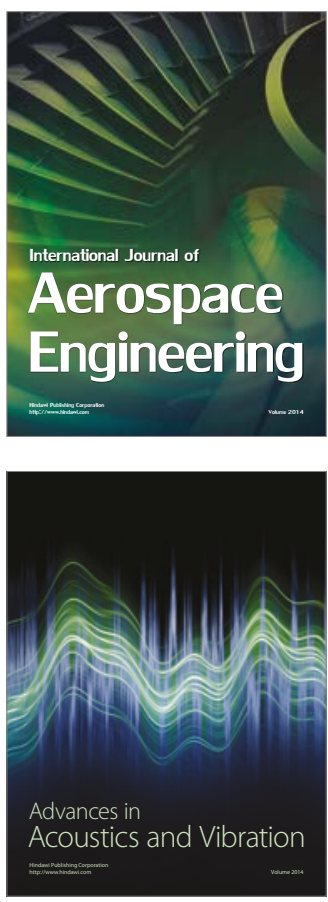

Sensor Networks 